\title{
Evidências de Validade da Versão Brasileira da Escala de Dimensões da Dependência do Tabaco (EDDT)
}

\author{
Germano Gabriel Lima Esteves ${ }^{1}$ \\ Universidade de Rio Verde, Rio Verde-GO, Brasil
}

\section{RESUMO}

Objetivou-se adaptar e reunir evidências de validade de construto e critério da Escala de Dimensões da Dependência do Tabaco (EDDT) para o contexto brasileiro. Participaram 270 fumantes ativos de diversos estados, sendo a maioria do sexo feminino $(51,7 \% ; f=139)$, com idade variando entre 18 à 68 anos $(M=33,77 ; D P=12,54)$ e com tempo de fumante variando de 1 a 53 anos $(M=15,01 ; D P=12,50)$. Primeiramente, foram realizados os procedimentos de back translation e validação de conteúdo. Após, os participantes responderam à Escala de Dimensões da Dependência do Tabaco (EDDT) e a um questionário sociodemográfico. A análise fatorial exploratória indicou uma estrutura composta por 26 itens divididos em quatro fatores. Também foram encontradas evidências de validade de critério para todos os fatores da EDDT em função da intenção de ingresso em um programa de dependência do tabaco. Como estudo inicial, a EDDT apresenta evidências de adequação psicométrica para avaliar a dependência do tabaco.

Palavras-chaves: dependência; tabagismo; validade; adaptação; psicometria.

\section{ABSTRACT - Validity evidence for the Brazilian version of the Tobacco Dependence Dimension Scale (TDDS)}

The aim was to adapt the Tobacco Dependence Dimension Scale (TDDS) for the Brazilian context and to gather evidence of construct and criterion validity. A total of 270 active smokers from different states participated, the majority being female $(51.7 \%$, $f=139)$, ranging in age from 18 to 68 years $(M=33.77, S D=12.54)$ and smoking time ranging from 1 to 53 years $(M=15.01$, $S D=12.50)$. First, back translation and content validation procedures were performed. Next, the participants responded to the Tobacco Dependence Dimension Scale (TDDS) and a sociodemographic questionnaire. Exploratory factor analysis indicated a structure composed of 26 items divided between four factors. Evidence of criterion validity was also found for all the TDDS factors as a function of intent to join a tobacco dependence program. According to this initial study, the TDDS presents evidence of psychometric adequacy to assess tobacco dependence.

Keywords: dependence; smoking; validity; adequacy; psychometrics.

RESUMEN - Evidencias de Validez de la Versión Brasileña de la Escala de Dimensiones de la Dependencia del Tabaco (EDDT)

Se ha objetivado adaptar y reunir evidencias de la validez del constructo y el criterio de la Escala de Dimensiones de la Adicción al Tabaco (EDAT) para el contexto brasileño. Han participado 270 fumadores activos de diversos estados, siendo la mayoría del género femenino $(51,7 \% ; f=139)$, con edad entre 18 y 68 años $(M=33,77 ; D S=12,54)$ y con tiempo de fumador variando entre 1 y 53 años $(M=15,01 ; D S=12,50)$. En primer lugar, se realizaron los procedimientos de back translation y la validación del contenido. A continuación, los participantes respondieron a la Escala de las Dimensiones de la Adicción al Tabaco (EDAT) y un cuestionario sociodemográfico. El análisis factorial exploratorio indicó una estructura compuesta por 26 ítems que se dividieron en cuatro factores. Del mismo modo, fueron encontradas evidencias de la validez de criterio para todos los factores de la EDAT en conformidad con la intención de ingreso en un programa de dependencia al tabaco. Como estudio inicial, la EDAT presenta evidencias de adecuación psicométrica para evaluar la dependencia al tabaco.

Palabras clave: dependencia; tabaquismo; validez; adaptación; psicometría.

No Brasil, desde o ano de 1980, o Ministério da Saúde, em parceria com o Instituto Nacional do Câncer (INCA), mantém o Programa Nacional de Controle do Tabagismo (PNCT) que tem como objetivo a prevenção da utilização do tabaco entre crianças e adolescentes, bem como oferecer tratamento para a redução do hábito de fumar entre dependentes de nicotina (INCA, 2014). Nesse contexto, a ênfase do modelo de tratamento adotado pelo Brasil apresenta-se preponderantemente focado no aspecto comportamental e farmacológico da dependência. $\mathrm{O}$ modelo consiste em uma consulta inicial em que o participante é avaliado quanto ao nível de

${ }^{1}$ Endereço para correspondência: Universidade de Rio Verde - UniRV. Laboratório de Avaliação Psicológica (LAPRV), Campus Rio Verde, Fazenda Fontes do Saber, 
dependência, por meio do Teste de Fagerström para a Dependência de Nicotina (FTND) que avalia os sintomas de dependência física (Fagerström, 1978), após recebe sessões de terapia, em grupo ou individual, focada em intervenções cognitivas e treinamento de habilidades comportamentais e, caso indicado, recebe o auxílio farmacológico (reposição de nicotina e bupropiona) (Mendes et al., 2016).

Entretanto, embora o auxílio farmacológico e a reposição de nicotina contribuam para redução dos sintomas da abstinência, alguns achados sugerem que o craving, entendido como um intenso desejo de utilizar a substância, pode ser resistente a intervenções farmacológicas e comportamentais (Schlagintweit \& Barrett, 2016). Assim, embora a adesão ao tratamento esteja em alta, o processo de recaída parece apresentar-se como exponencial, ou seja, um grande percentual de indivíduos que recebem tratamento acaba por retornar ao hábito de fumar com o passar do tempo. As taxas de recaída apontadas por estudos de meta-análise, que combinaram o efeito de diversas intervenções para fumantes, indicam que, por volta dos seis meses após o tratamento, uma taxa de cerca de $70 \%$ dos indivíduos que receberam a intervenção pode recair, chegando a cerca de 80\% após 12 meses (Pawlina, Rondina, Espinosa \& Botelho, 2016). Além disso, chama a atenção as altas taxas de abandono durante o tratamento que geram uma perda anual de aproximadamente 338,6 milhões reais (Pawlina et al., 2016). Apesar da dificuldade de manutenção na eficiência do tratamento, o índice de sucesso no abandono do hábito com foco no desejo ou intenção de parar de fumar e sem a participação de programas para tratamento do tabagismo também não se oferece como uma alternativa viável, apresentando um percentual de apenas $5 \%$ de sucesso no abandono do hábito (APA, 1996).

De tal modo, aponta-se aqui que o estabelecimento de um plano terapêutico deveria abordar fatores além da dependência física, como apoio social e fatores sociodemográficos (Campos \& Gomide, 2015; França et al., 2015). Corrobora com essa proposição dois estudos realizados por Piasecki, Kenford, Smith, Fiore e Baker (1997), que apontaram a falta de contribuição para validade incremental de marcadores da dependência física na recaída do paciente e a existência de trajetórias diferentes dos sintomas de abstinência e vulnerabilidade no processo de recaída, sugerindo que o reconhecimento de novos aspectos da dependência poderá aumentar o poder explicativo e contribuir para novas estratégias.

Compreende-se, portanto, que o enfoque comportamental e farmacológico adotado pelo PNCT, desde a sua avaliação inicial, deixa de incluir outros aspectos importantes da dependência do tabaco, que podem estar contribuindo com a ineficácia da manutenção da abstinência, como: 1. a dimensão social, compreendida como a utilização do tabaco como facilitador social, induzindo a um senso de pertencimento de determinado grupo;
2. a dimensão emocional, que envolve a utilização do tabaco como forma de controle dos sentimentos, como ansiedade, tédio, estresse e raiva e; 3 . a dimensão sensorial, que refere-se a sensação de prazer e bem-estar relacionada a nicotina (liberação de dopamina) (Campos \& Gomide, 2015; Kim, Ko, Kim, Kim \& Kim, 2015). Nesse contexto, a proposição de um instrumento psicometricamente adequado e que possa mensurar tais aspectos pode contribuir para o melhor entendimento da influência desses fatores no processo de recaída.

\section{Dependência do tabaco}

$\mathrm{Na}$ literatura, os aspectos social, sensorial, comportamental e emocional da dependência do tabaco são contemplados pelo modelo descrito por Russell (1971), que engloba três estágios da dependência: 1 . inicialmente, no primeiro estágio, o indivíduo se sente motivado a utilização do cigarro por fatores psicossociais, como a necessidade de aceitação e pertença grupal (dependência social); 2. no segundo estágio, a dependência sensorial ocorre por meio da nicotina em baixas quantidades que estimula a liberação de neurotransmissores acetilcolina, glutamato, dopamina, serotonina e ácido gama-aminobutírico (GABA) promovendo um aumento no estado de alerta, redução do apetite, melhora na atenção e concentração, produzindo efeitos semelhantes àqueles descritos por usuários de crack e/ou cocaína (APA, 1996). Assim, do mesmo modo que outras substâncias que causam dependência, a partir da ação nas vias dopaminérgicas do sistema mesolímbico, a nicotina induz a liberação de dopamina no nucleus accumbens, que estimula a sensação de prazer e recompensa e diminui a atividade talâmica (Brennan, Lea, Fitzmaurice, \& Truman, 2010). Assim, o indivíduo passa a experimentar os efeitos reforçadores do tabaco, como a sensação de alerta e relaxamento, gerando uma dependência sensorial; 3. já no terceiro estágio, o uso contínuo da nicotina leva ao desenvolvimento de tolerância e dependência química da substância, fazendo com que o indivíduo necessite de uma maior quantidade da substância para não experimentar os sintomas da abstinência (Planeta \& Cruz, 2005). A síndrome de abstinência inclui craving, ansiedade, irritabilidade, aumento no apetite, insônia e desregulação do humor (Cosci, Bertoli, Pistelli, \& Carrozzi, 2016). Desse modo, o terceiro estágio engloba a dependência física (sintomas comportamentais da abstinência) e dependência emocional (utilização do tabaco para regulação do humor). Diferentemente da dependência psicológica, que abrange o condicionamento e associações do hábito de fumar (ex.: fumar tomando café, fumar e dirigir, fumar após refeições), a dependência emocional está relacionada apenas com à utilização do tabaco para regulação emocional (ex.: redução do estresse, raiva, ansiedade) (Johnson et al., 2005; Santos \& Costa, 2015).

No Brasil, a maioria dos estudos sobre dependência do tabaco tem negligenciado que o problema 
da dependência do tabaco é multidimensional, ou seja, as pessoas são influenciadas por fatores demográficos, como sexo e idade; fatores sociais, como a utilização do tabaco para o pertencimento de determinado grupo; fatores emocionais, como a utilização do tabaco para regulação do estresse e ansiedade; fatores sensoriais, como a sensação de prazer e bem-estar relacionada à nicotina (liberação de dopamina) e fatores comportamentais, como a utilização do tabaco para redução dos sintomas de abstinência (Campos \& Gomide, 2015; Johnson et al., 2005). Acredita-se que um dos motivos para a escassez de estudos abordando esses aspectos, apesar da disponibilidade de instrumentos, é a inexistência de instrumentos adaptados e com parâmetros psicométricos adequados que englobem os aspectos sociais, emocionais e sensoriais na avaliação da dependência do tabaco (Santos, Silveira, Oliveira \& Ciaffa, 2011).

Já no âmbito internacional, Johnson et al. (2005) desenvolveram um instrumento baseado na natureza multidimensional da dependência do tabaco. A Dimensions of Tobacco-Dependece Scale (DTDS), traduzida como Escala de Dimensões da Dependência do Tabaco (EDDT), composta por 54 itens em sua versão original e, posteriormente, reduzida para 27 itens de acordo com critérios de validade de conteúdo e discriminação dos itens (Johnson et al., 2005; Richardson et al., 2007). Os 27 itens apresentam-se distribuídos em quatro dimensões: dependência social (composta por seis itens) $(\alpha=0,89)$, que diz respeito à estrutura social, como a manutenção de relacionamento com amigos e sentimento de pertença social em determinados grupos; dependência sensorial (composta por cinco itens) $(\alpha=0,72)$, apresentada como as sensações positivas relacionadas ao hábito de utilização do cigarro, como o sabor e o efeito da fumaça tragada; dependência emocional (composta por seis itens) $(\alpha=0,90)$, relacionada ao controle ou gerenciamento de emoções por meio da manutenção do hábito de fumar; e a dependência física (composta por 10 itens) $(\alpha=0,96)$, configurada como manutenção do hábito de fumar para controle dos sintomas de abstinência (Johnson et al., 2005; Richardson et al., 2007). Ademais, a escala apresenta boa adequação na sua validação coreana, realizada por Kim et al. (2015), com alfas de Cronbach superiores a 0,80 para todos os fatores.

Portanto, a adaptação da EDDT possibilita a avaliação dos parâmetros psicométricos de uma medida multidimensional para a dependência do tabaco, inexistente no Brasil, apresentando um instrumento que pode ser empregado em pesquisas e contribuir na elaboração de estratégias de intervenção voltadas para a dependência do tabaco. Diante disso, a presente pesquisa objetivou adaptar e reunir evidências de validade de estrutura interna e de validade baseada na relação com variáveis externas (critério por grupos contrastantes) da Escala de Dimensões da Dependência do Tabaco (EDDT) para o contexto brasileiro.

\section{Método}

\section{Delineamento}

Trata-se de uma pesquisa transversal com delineamento ex-post-facto e de comparação entre participantes.

\section{Participantes}

Contou-se com uma amostra não probabilística, isto é, de conveniência, de 270 participantes que se autodeclararam fumantes ativos com, no mínimo, um ano de uso do cigarro, de setes estados (AL, PB, GO, $\mathrm{BA}, \mathrm{SE}, \mathrm{DF}$ e PE), sendo a maioria do sexo feminino $(51,7 \% ; f=139)$, com idade variando ente 18 à 68 anos $(M=33,77 ; D P=12,54)$ e com tempo de fumante variando de 1 a $53(M=15,01 ; D P=12,50)$.

\section{Instrumentos}

Para a coleta de dados, os participantes foram solicitados a responder dois instrumentos de pesquisa descritos a seguir por meio de survey eletrônico.

Questionário sociodemográfico. Constituído por dados, como sexo, idade, tempo de utilização de cigarro a base de tabaco e uma pergunta critério sobre a intenção de redução de uso do cigarro. Assim, foi perguntado aos participantes se eles teriam a interesse em ingressar em algum programa de dependência do tabaco nos próximos seis meses, que responderam sim ou não.

Escala de Dimensões da Dependência do Tabaco (EDDT). Desenvolvida por Johnson et al. (2005) e reduzida por Richardson et al. (2007). Ao final da etapa de tradução reversa e validação de conteúdo, contou-se com a Escala de Dependência do Tabaco composta por 27 itens respondidos em uma escala de quatro pontos, "1= não me descreve em absoluto" até " $4=$ descreve-me totalmente".

\section{Procedimentos}

Inicialmente, o projeto foi submetido ao Comitê de Ética em Pesquisa (CEP) por meio da Plataforma Brasil, tendo sido aprovado (CAAE: 1.955.337), a aplicação foi realizada por meio do questionário eletrônico divulgado em redes sociais. Os participantes foram esclarecidos do caráter voluntário e sigiloso, bem como o respeito às diretrizes éticas que regem a pesquisa com seres humanos e, após, obteve-se o consentimento livre e esclarecido de todos os participantes que se voluntariaram. Para a seleção da amostra, os participantes, após o aceite do TCLE, eram conduzidos a uma segunda tela onde era solicitado o preenchimento da presença ou ausência dos critérios de inclusão: 1. presença do hábito de fumar nos últimos 12 meses, 2. não participação em programas de dependência; e critérios de exclusão: 1. fumar cigarros que não fossem a base de tabaco, 2. utilizar algum medicamento para dependência ou abstinência. Assim, aqueles indivíduos que não atendiam ao perfil desejado eram redirecionados para uma última tela que agradecia o interesse 
em participar da pesquisa, mas que, naquele momento ele não apresentava o perfil estudado. Desse modo, apenas os sujeitos que atendiam os critérios de inclusão tiveram acesso à página em que os instrumentos de pesquisa estavam disponíveis.

\section{Procedimentos de tradução}

Para a tradução da escala, adotou-se o procedimento de back translation (tradução reversa). Assim, em uma primeira etapa, os itens da escala foram traduzidos para o português por três tradutores bilíngues, com formação em Psicologia. Posteriormente, em uma segunda etapa, as três versões foram retraduzidas, do português para o inglês, por outros três tradutores que não tiveram contato prévio com o instrumento. Por fim, em uma terceira etapa, foi realizada a consolidação da versão preliminar. Em posse das seis versões (três em português e três em inglês), um comitê formado por três pesquisadores (um doutor e dois mestres em Psicologia) avaliou a proximidade de cada item da versão original com as versões dos itens traduzidos. Assim, quando a retradução, o item era exatamente igual ao original, era aprovado na sua versão traduzida. Quando a retradução do item divergia do original, o item era discutido, levando em conta a definição do construto, até um acordo entre os integrantes do comitê de qual seria a melhor tradução. Desse modo, foi comporta a versão preliminar da EDDT em português.

\section{Procedimentos de validação de conteúdo}

Com o intuito de verificar a pertinência teórica, prática e a clareza na linguagem dos itens da Escala de Dimensões da Dependência do Tabaco (EDDT), procedeu-se a validação do conteúdo. Para tanto, contou-se com a participação de quatro juízes (todos possuindo formação em Psicologia, sendo dois com experiência em psicometria e dois com experiência em dependência química) que responderam em uma escala de cinco pontos, variando de 1 "muito pouco pertinente/claro" a 5 "totalmente pertinente/claro", sobre a pertinência teórica, prática e a clareza na linguagem de cada item. Com as respostas, foram calculados os coeficientes de validade de conteúdo (CVC) para todos os aspectos, de acordo com Hernández-Nieto (2002), para cada item, mantendo aqueles que obtiveram CVC $>0,80$, e para toda a escala, que se demonstraram satisfatórios, a saber: pertinência teórica $=0,90$; pertinência prática $=0,95$; clareza de linguagem $=0,97$.

Durante a validação do conteúdo, no critério de clareza da linguagem, os juízes avaliadores sugeririam mudanças no conteúdo de um item, de modo a adequá-los para a população adulta. Desse modo, optou-se pelo termo "meio social" em detrimento de "escola".

\section{Análise de dados}

As análises foram realizadas por meio do Statistical Package for the Social Sciences (SPSS) versão 24. Em um primeiro momento, foram realizadas estatísticas descritivas (média e desvio padrão) com as respostas do questionário sociodemográfico no intuito de apresentar um perfil amostral.

Inicialmente, foi verificado se os dados satisfaziam os pressupostos da análise fatorial exploratória (AFE). Nesse contexto, como os dados foram coletados via questionário eletrônico e houve a obrigatoriedade de resposta para cada item (caso o respondente não marcasse um ponto na escala o questionário não prosseguia para página seguinte) não houveram casos ausentes. Para análise da normalidade, foram realizadas análises da skewness e curtose de cada item e realizados testes Kolmogorov-Smirnov, os resultados indicaram a normalidade das variáveis. Também foi verificada a linearidade e não foi verificada a presença de outliers. Por fim, verificou-se a multicolinearidade por meio da matriz de correlações.

Com os pressupostos satisfeitos, foi realizada uma AFE, seguindo os mesmos procedimentos adotados na versão original da escala, utilizando-se a técnica de Máxima Verossimilhança com o método de rotação oblíqua promax. Adotou-se também um valor mínimo de saturação do item considerado aceitável de 0,30. A confiabilidade dos fatores da escala foi realizada por meio da técnica de alfa de Cronbach $(\alpha)$. Por fim, a validade de critério da EDDT foi analisada por meio do teste $\mathrm{t}$ de Student, para se verificar a existência de diferenças nos escores médios dos fatores da EDDT em função da intenção de ingresso em um programa de dependência do tabaco.

\section{Resultados}

\section{Análise fatorial exploratória}

Inicialmente, constatou-se a fatorabilidade da matriz por meio do teste Kaiser-Meyer-Olkin (KMO), que se demonstrou aceitável $(0,89)$, e do teste de esfericidade de Bartlett $\left(\chi^{2}(351)=4263,439 ; p \leq 0,000\right)$. O critério de Kaiser-Guttman foi utilizado para retenção de fatores e foi obtido um modelo de quatro fatores, condizentes com a estrutura teórica, com eigenvalues de 9,20; 3,77; 1,68 e 1,50. O mesmo foi observado na análise gráfica do scree plot, que corroborou com a extração de quatro fatores (Damásio, 2012).

Com base nesses resultados descritos, procedeu-se à AFE, com o método de rotação promax, fixando a extração de quatro fatores. O resultado dessa análise pode ser visualizado na Tabela 1, onde são apresentadas as cargas fatoriais dos itens nos seus respectivos fatores, a variância explicada e os índices de consistência interna para cada fator.

A AFE indicou que o item 16 ("Mesmo quando eu não tenho tempo para um cigarro inteiro eu consigo me saciar com poucos tragos.") apresentou saturação abaixo de 0,30, sendo consequentemente eliminado. Já o item 24 ("Fumar faz com que coisas, como tomar um 
refrigerante ou um café, sejam mais agradáveis.”), que na escala original apresentou maior carga fatorial no fator "Dependência sensorial", neste estudo apresentou uma carga fatorial inferior a 0,30 neste fator e uma carga fatorial de 0,56 no fator "Dependência física". Assim, optou-se por reter o item no fator com maior carga fatorial.

Tabela 1

Cargas Fatoriais dos Itens da Escala de Dimensões da Dependência do Tabaco (EDDT)

\begin{tabular}{|c|c|c|c|c|c|}
\hline \multirow{2}{*}{$\mathrm{N}^{\circ}$} & \multirow{2}{*}{ Item } & \multicolumn{4}{|c|}{ Fatores } \\
\hline & & F1 & F2 & F3 & F4 \\
\hline 12. & Sinto-me ansioso para o meu próximo cigarro. & 0,89 & & & \\
\hline 26. & Meu corpo precisa de cigarros para se sentir bem. & 0,86 & & & \\
\hline & Eu tenho fortes desejos de fumar cigarros. & 0,82 & & & \\
\hline & Meu corpo implora por cigarros quando eu não fumo. & 0,80 & & & \\
\hline 07. & Eu posso funcionar melhor depois do meu primeiro cigarro do dia. & 0,80 & & & \\
\hline & Eu preciso manter meus níveis de nicotina elevados. & 0,71 & & & \\
\hline 14. & Sinto-me em pânico quando fico sem cigarros. & 0,65 & & & \\
\hline 10. & Eu me concentro melhor depois de um cigarro. & 0,63 & & & \\
\hline & Meu ato de fumar é automático. Eu nem sequer penso nisso. & 0,63 & & & \\
\hline & Fumar faz com que coisas. como tomar um refrigerante ou um café, sejam mais agradáveis. & 0,56 & & & \\
\hline 11. & Eu preciso de um cigarro para me acalmar quando eu estou zangado. & & 0,93 & & \\
\hline 19. & Eu preciso fumar quando estou nervoso. & & 0,88 & & \\
\hline 22. & Eu preciso fumar quando estou deprimido. & & 0,83 & & \\
\hline 02. & Eu preciso fumar quando estou estressado. & & 0,77 & & \\
\hline 06. & Eu preciso fumar quando estou triste. & & 0,70 & & \\
\hline 25. & Eu preciso fumar para relaxar. & & 0,53 & & \\
\hline 15. & Fumar me faz sentir popular. & & & 0,81 & \\
\hline 21. & Fumar me faz parecer legal. & & & 0,79 & \\
\hline 18. & Dar cigarros aos meus amigos me faz sentir importante. & & & 0,70 & \\
\hline 08. & Fumar me faz parecer mais maduro. & & & 0,69 & \\
\hline 27. & Compartilhar cigarros me ajuda a me sentir mais próximo das outras pessoas. & & & 0,69 & \\
\hline 01. & Fumar me ajuda a ser aceito no meio social. & & & 0,57 & \\
\hline 04. & Eu gosto da sensação de soprar a fumaça. & & & & 0,82 \\
\hline 17. & Eu curto segurar e manusear cigarros. & & & & 0,71 \\
\hline 09. & Eu gosto do sabor dos cigarros. & & & & 0,67 \\
\hline 13. & Eu aprecio a sensação de fumaça em meus pulmões. & & & & 0,62 \\
\hline Eige & nvalues & 9,20 & 3,77 & 1,68 & 1,50 \\
\hline \multicolumn{6}{|c|}{$\mathrm{KMO}=0,89$} \\
\hline \multicolumn{6}{|c|}{ \%variância explicada $=59,88$} \\
\hline Alfa & $(\alpha)$ & 0,92 & 0,93 & 0,81 & 0,71 \\
\hline
\end{tabular}

Além disso, a AFE apontou que o fator 1 (eigenvalues $=9,20$ ), responsável por $34,08 \%$ da variação total e denominado de "dependência física", é composto por um total de 10 itens $(03,05,07,10,12,14,20,23$, $24,26)$ com cargas fatoriais variando entre 0,89 (item 12) até 0,56 (Item 24). A análise do conteúdo dos itens desse fator indica que estes dizem respeito a situações que incluem craving, resistência e sintomas de abstinência.

O segundo fator (eigenvalues $=3,77$ ), responsável por $13,97 \%$ da variação total, foi composto por um total de seis itens $(02,06,11,19,22,25)$ com cargas fatoriais variando entre 0,93 (item 11) até 0,53 (item 25). Esse fator, denominado "dependência emocional", agrupa itens que estão relacionados ao controle, recompensa e gerenciamento emocional e gerenciamento do estresse por meio da utilização do cigarro.

No terceiro fator (eigenvalues $=1,68$ ), responsável por $6,24 \%$ da variação total, foram retidos seis itens $(01,08$, $15,18,21,27)$ com cargas fatoriais variando entre 0,81 (item 15) até 0,57 (item 01). A leitura dos itens desse fator indica que ele se refere à "dependência social", abrangendo elementos como a aceitação social, identificação 
entre pares de grupo por meio do compartilhamento de cigarros e indicativos de manutenção de relações sociais, pessoais por meio da utilização do cigarro.

Já o quarto fator (eigenvalues=1,50), nomeado de "dependência sensorial" e responsável por $5,57 \%$ da variação total, foi composto por quatro itens $(04,09,13$, 17) com cargas fatoriais variando entre 0,82 (item 04) até 0,62 (item 13). Esse fator envolve itens relacionados às sensações positivas relacionadas ao hábito de utilização do cigarro, como o sabor e o efeito da fumaça tragada.

No tocante à análise de confiabilidade, os fatores apresentaram boa consistência interna, a saber: dependência física $(\alpha=0,92)$, dependência emocional $(\alpha=0,93)$, dependência social $(\alpha=0,81)$ e dependência sensorial $(\alpha=0,71)$.

\section{Validade de critério}

Os resultados da validade de critério também se apresentaram em consonância com o modelo proposto por Russell (1971), que reporta os fatores de dependência social e sensorial como aspectos iniciais do desenvolvimento da dependência do tabaco e associados às sensações positivas derivadas da nicotina. Assim, indivíduos com maiores níveis de dependência social e sensorial tendem a reportar um desinteresse em participar de um programa de dependência. Em contraponto, os fatores físicos e emocionais são aspetos de uma dependência do tabaco já instalada e estão associados aos sintomas de abstinência, como vômito, febre, ansiedade e irritabilidade (Halty, Hüttner, Oliveira, Santos \& Martins, 2002). De tal modo, indivíduos com maiores níveis de dependência física e emocional tendem a indicar interesse em participar de um programa de dependência.

Nesse contexto, esperava-se que, nos fatores de dependência física e dependência emocional, aqueles participantes que declararam ter interesse em ingressar em algum programa de dependência do tabaco nos próximos seis meses reportasse uma média superior e significativamente diferente daqueles participantes que declararam não ter o interesse. De modo contrário, nos fatores de dependência social e sensorial, esperava-se que os participantes que declaram não ter o interesse em ingressar em algum programa de dependência do tabaco nos próximos seis meses reportasse uma média superior e significativamente diferente daqueles participantes que declararam ter o interesse.

Tabela 2

Diferenças entre os Fatores da EDDT em Função do Interesse de Participação do Programa de Dependência

\begin{tabular}{|c|c|c|c|c|c|c|c|c|c|c|c|}
\hline & \multirow{2}{*}{ Grupo } & \multirow{2}{*}{ M } & \multirow{2}{*}{$D P$} & \multirow{2}{*}{$E P$} & \multirow{2}{*}{$t$} & \multirow{2}{*}{ gl } & \multirow{2}{*}{$p$} & \multirow{2}{*}{$\mathrm{DM}$} & \multirow{2}{*}{$d$} & \multicolumn{2}{|c|}{ IC de $95 \%$} \\
\hline & & & & & & & & & & LI & LS \\
\hline \multirow{2}{*}{ Física } & Sim & 2,99 & 0,91 & 0,06 & \multirow{2}{*}{3,04} & \multirow{2}{*}{267} & \multirow{2}{*}{0,00} & \multirow{2}{*}{0,35} & \multirow{2}{*}{0,40} & \multirow{2}{*}{0,12} & \multirow{2}{*}{0,59} \\
\hline & Não & 2,63 & 0,86 & 0,09 & & & & & & & \\
\hline \multirow{2}{*}{ Emocional } & Sim & 3,44 & 0,82 & 0,06 & \multirow{2}{*}{2,26} & \multirow{2}{*}{266} & \multirow{2}{*}{0,02} & \multirow{2}{*}{0,25} & \multirow{2}{*}{0,29} & \multirow{2}{*}{0,32} & \multirow{2}{*}{0,47} \\
\hline & Não & 3,19 & 0,92 & 0,09 & & & & & & & \\
\hline \multirow{2}{*}{ Social } & Sim & 1,38 & 0,53 & 0,03 & \multirow{2}{*}{$-2,55$} & \multirow{2}{*}{266} & \multirow{2}{*}{0,01} & \multirow{2}{*}{$-0,23$} & \multirow{2}{*}{0,38} & \multirow{2}{*}{$-0,40$} & \multirow{2}{*}{$-0,52$} \\
\hline & Não & 1,61 & 0,75 & 0,08 & & & & & & & \\
\hline \multirow{2}{*}{ Sensorial } & Sim & 2,19 & 0,84 & 0,06 & \multirow{2}{*}{$-4,98$} & \multirow{2}{*}{267} & \multirow{2}{*}{0,00} & 0 & 5 & דר & קר 2 \\
\hline & Não & 2,74 & 0,84 & 0,09 & & & & $-0,55$ & 0,65 & $-0,1 /$ & $-0,33$ \\
\hline
\end{tabular}

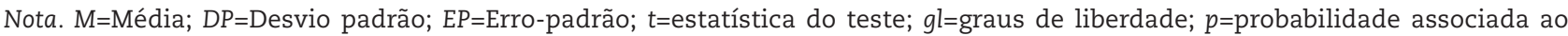
resultado; $D M=$ diferença das médias dos grupos; $d=$ tamanho do efeito; LI=Limite inferior do intervalo de confiança de $95 \%$; LS=Limite Superior do intervalo de confiança de $95 \%$

Como pode-se observar na Tabela 2, o teste $t$ indicou que aqueles participantes que se percebem com um maior nível de dependência física e emocional do tabaco reportaram ter interesse em participar de um programa de dependência, com uma diferença média de 0,35 (IC95\% 0,12-0,59) e 0,25 (IC95\% 0,32-0,47), respectivamente, daqueles que indicaram não ter o interesse, com um tamanho de efeito mediano 0,40 e 0,29 , respectivamente - Cohen (1992) indica que, para o tamanho do efeito do teste $t, 0,20$ é um efeito pequeno, 0,50 é um efeito médio e 0,80 é um efeito grande. Em contraponto, os participantes que se percebem com um maior nível de dependência social e sensorial reportaram não ter o interesse, com uma diferença média de $-0,23$ [IC95\% $-0,40$, $-0,52]$ e $-0,55$ [IC95\% - $0,77,-0,33]$, respectivamente, daqueles que indicaram não ter o interesse, com um tamanho de efeito mediano 0,38 e 0,65 , respectivamente.

\section{Discussão}

O resultado da análise fatorial indicou um modelo de quatro fatores. Esse modelo apresenta-se em 
consonância com o instrumento original proposto por Johnson et al. (2005) que contou com a participação de 513 adolescentes fumantes. Apesar de o instrumento original apresentar-se voltado para uma população de adolescentes, o modelo teórico de base, proposto por Russell (1971), não se restringe a esse perfil. Assim, a adaptação do instrumento realizada na presente pesquisa reuniu evidências de adequação também para população adulta, indicando que outros fatores devem ser levados em conta na avaliação da dependência do tabaco.

No tocante a quantidade de itens, nesta pesquisa, foi encontrada diferença quando em comparação com os estudos de validação do instrumento original e em outros países. Na amostra estudada, a EDDT em sua versão final apresenta 26 itens, sendo o item 16 ("Mesmo quando eu não tenho tempo para um cigarro inteiro eu consigo me saciar com poucos tragos.") excluído por conta da baixa carga fatorial $(0,30)$. Acreditase que essa discrepância possa ser explicada pelo perfil da amostra em algumas variáveis, como o tempo de utilização do cigarro médio que é maior do que o reportado por adolescentes.

Em geral, as propriedades psicométricas da EDDT reportadas nesta pesquisa podem ser consideradas satisfatórias. Os fatores apresentaram boa consistência interna (dependência física $\alpha=0,92)$; emocional $(\alpha=0,93)$; social $(\alpha=0,81)$ e sensorial $(\alpha=0,71)$ e semelhantes aos encontrados no estudo de validação da escala original realizado por Johnson et al. (2005), que reportou os seguintes alfas de Cronbach: $(\alpha=0,96)$ para dependência física; $(\alpha=0,90)$ para dependência emocional; $(\alpha=0,89)$ para dependência social; e $(\alpha=0,72)$ para dependência sensorial. Esses resultados também coadunam com a validação coreana da EDDT (Kim et al., 2015), que indicaram valores de alfas de: 0,91 para dependência física; $(\alpha=0,92)$ para dependência emocional; $(\alpha=0,88)$ para dependência social e; $(\alpha=0,86)$ para dependência sensorial.

Com relação aos resultados das evidências de validade baseada na relação com variáveis externas (critério por grupos contrastantes), foram apresentados dados que corroboram com o modelo proposto por Russell (1971). Tais evidências indicam que o instrumento apresenta uma sensibilidade na distinção de pacientes que terão maior probabilidade de se engajar no tratamento. Desse modo, o instrumento pode ajudar na identificação e encaminhamento de pacientes para intervenções que tenham como foco o tipo de dependência predominante no paciente. Em termos práticos, a EDDT providencia uma avaliação que abrange os fatores relacionados à dependência do tabaco. A disponibilidade de um instrumento psicometricamente adequado para avaliação desses fatores implica diretamente no processo de triagem para programas antitabagismo, que pode adotar estratégias de intervenções por grupos, de acordo com o nível dos fatores de dependência mais presente no indivíduo. Assim, a intervenção poderá ser otimizada evitando que o usuário se integre ao programa antitabagismo recebendo um auxílio focado em fatores que pouco influenciam na sua dependência. A longo prazo, a utilização desse instrumento na prática pode utilizar a redução do número de pacientes que irão recair.

\section{Considerações finais}

O presente estudo objetivou adaptar e reunir evidências de validade de estrutura interna e de validade baseada na relação com variáveis externas (critério por grupos contrastantes) da Escala de Dimensões da Dependência do Tabaco (EDDT) para o contexto brasileiro. Assim, procurou-se apresentar uma medida de avaliação das dimensões física, emocional, social e sensorial da dependência do tabaco, viabilizando a avaliação desses fatores em pesquisas futuras, triagens e planejamento de intervenções antitabagismo.

É importante destacar que a coleta de dados da presente pesquisa ocorreu via redes sociais e que o link do questionário eletrônico foi compartilhado pelos autores do estudo, integrantes do grupo de pesquisa e em grupos de fumantes. Assim, o contexto específico da amostra pode ter acarretado algum viés não investigado. Entretanto, é de grande importância que o funcionamento desses grupos (postagens, compartilhamento de experiências e likes) sejam avaliados para identificação desse mecanismo que pode ser visto como uma rede de apoio.

No entanto, os resultados devem ser interpretados com moderação, pois vale destacar a necessidade de mais evidências da adequação psicométrica da escala por meio de outros métodos, como buscar evidências de validade por meio da relação dos escores da EDDT e o teste de Fagerström. Nesse contexto, por ter sido este o primeiro estudo que buscou adaptar e investigar evidências de validade da EDDT no Brasil, entende-se que a estrutura apresentada é a mais adequada, mas é provável que outros estudos agreguem maior contribuição na variância comum dos seus fatores correspondentes. Por fim, sugere-se que pesquisas futuras procurem ampliar a amostra e verificar interação dos fatores da EDDT com algumas variáveis demográficas, como sexo, idade e tempo de utilização do cigarro, fatores que aparecem associados ao abandono do tratamento. Além disso, a confiabilidade da EDDT pode ser avaliada por outros métodos, como teste-reteste, e, também, estudos futuros poderão estabelecer normas para uma melhor interpretação dos resultados. 


\section{Referências}

American Psychiatric Association. (1996). Practice guideline for the treatment of patients with nicotine dependence. American Journal of Psychiatry, 153, 1-31. doi: 10.1176/ajp.153.10.1

Brennan, K. A., Lea, R. A., Fitzmaurice, P. S., \& Truman, P. (2010). Nicotinic receptors and stages of nicotine dependence. Journal of Psychopharmacolgy, 24(6), 793-808. doi: 10.1177/0269881108100256

Campos, P. C. M., \& Gomide, M. (2015). O Programa Nacional de Controle do Tabagismo (PNCT) na perspectiva social: A análise de redes, capital e apoio social. Caderno de Saúde Coletiva, 23(4), 436-444. doi: 10.1590/1414-462X201500040241

Cohen, J. (1992). A power primer. Psychological bulletin, 112(1), 155.

Cosci, F., Bertoli, G., Pistelli, F., \& Carrozzi, L. (2016). Depressive, anxious, withdrawal symptoms, and craving as possible predictors of abstinence maintenance in smokers attending a 12-week quitting program. MedicalExpress, 3(1). doi: 10.5935

Damásio, B. F. (2012). Uso da análise fatorial exploratória em psicologia. Avaliação Psicologica: Interamerican Journal of Psychological Assessment, 11(2), 213-228. Recuperado de http://pepsic.bvsalud.org/scielo.php?script=sci_abstract\&pid=S1677-04712012000200007

Fagerström K. O. (1978). Measuring degree of physical dependence to tobacco smoking with reference to individualization of treatment. Addicive Behaviors, 3, 235-41. doi: 10.1016/0306-4603(78)90024-2

França, S. A. de S., Neves, A. L. F., Souza, T. A. S., Martins, N. C. N., Carneiro, S. R., Sarges, E. S. N. F., \& Souza, M. F. A. H. (2015). Fatores associados à cessação do tabagismo. Revista de Saúde Pública, 49, 10. doi: 10.1590/S0034-8910.2015049004946

Halty, L. S., Hüttner, M. D., Oliveira N. I. C., Santos, V., \& Martins, G. (2002). Análise da utilização do Questionário de Tolerância de Fagerström (QTF) como instrumento de medida da dependência nicotínica. Jornal de Pneumologia, 28(4), 180-186. doi: 10.1590/s010235862002000400002

Hernández-Nieto, R. A. (2002). Contributions to Statistical Analysis (pp. 119). Mérida: Universidad de Los Andes.

Instituto Nacional de Câncer. (2014). Programa Nacional de Controle do Tabagismo. Rio de Janeiro, INCA. Recuperado de http://www2.inca. gov.br/wps/wcm/connect/acoes_programas/site/ home/nobrasil/programa-nacional-controle-tabagismo

Johnson, J. L., Ratner, P. A., Tucker, R. S., Bottortt, J. L., Zumbo, B., Prkachin, K. M., \& Shoveller, J. (2005). Development of a multidimensional measure of tobacco dependence in adolescence. Addicive Behaviors, 30(3), 501-505. doi: 10.1016/j.addbeh.2004.07.006

Kim, J. Y., Ko, S. H., Kim, H. K., Kim, S. R., \& Kim, H. Y. (2015). Reliability and Validity of the Korean Version of the Dimensions of Tobacco Dependence Scale for Adolescents. Asia-Pacific Journal of Public Health, 27(6), 661-669. doi: 10.1177/1010539515583504

Mendes, A. C. R., Toscano, C. M., Barcellos, R. M. S., Ribeiro, A. L. P., Ritzel, J. B., Cunha, V. S., \& Duncan, B. B. (2016). Costs of the Smoking Cessation Program in Brazil. Revista de Saúde Pública, 50(10). doi: 10.1590/S1518-8787.2016050006303

Pawlina, M. M. C., Rondina, R. C., Espinosa, M. M., \& Botelho, C. (2016). Abandonment of nicotine dependence treatment: A cohort study. São Paulo Med. Jornal, 134(1), 47-55. doi: 10.1590/1516-3180.2015.00830309

Piasecki, M. T., Kenford, S. L., Smith, S. S., Fiore, M. C., \& Baker, T. B. (1997). Listening to nicotine: Negative affect and the smoking withdrawal conundrum. Psychological Science, 8(3), 184-189. doi: 10.1111/j.1467-9280.1997.tb00409.x

Planeta, C. S., \& Cruz, F. C. (2005). Bases neurofisiológicas da dependência do tabaco. Archives of Clinical Psychiatry, 32(5), 251-258. doi: 10.1590/S0101-60832005000500002

Richardson, C. G., Johnson, J. L., Ratner, P. A., Zumbo, B. D., Bottorff, J. L., Shoveller, J. A., \& Prkachin, K. M. (2007). Validation of the Dimensions of Tobacco Dependence Scale for adolescents. Addictive Behaviors, 32, 1498-1504. doi: 10.1016/j.addbeh.2006.11.002

Russell, M. A. H. (1971). Cigarette smoking: Natural history of a dependence disorder. British Journal of Medical Psychology, 44, 1-16. doi: 10.1111/j.2044-8341.1971.tb02141

Santos, N. F., \& Costa, R. A. (2015). Parental Tobacco consumption and child development. Jornal de pediatria, 91(4), 366-372. doi: 10.1016/j. jped.2014.09.006

Santos, J. D. P., Silveira, D. V., Oliveira, D. F., \& Caiaffa, W. T. (2011). Instrumentos para avaliação do tabagismo: Uma revisão sistemática. Ciência \& Saúde Coletiva, 16(12), 4707-4720. doi: 10.1590/S1413-81232011001300020

Schlagintweit, H. E., \& Barrett, S. P. B. (2016). Does acute tobacco smoking prevent cue-induced craving? Jornal of psychopharmacology, 30(5), 468-473. doi: $10.1177 / 0269881116639288$

\section{Sobre o autor}

Germano Gabriel Lima Esteves é doutorando em Psicologia Social, do Trabalho e das Organizações na Universidade de Brasília (PSTO/UnB). Atualmente, é Professor no Departamento de Psicologia da Universidade de Rio Verde, campus Rio verde, e coordenador do Laboratório de Avaliação Psicológica de Rio Verde (LAP-RV). 